\title{
DIFFERENTIAL IMPACT OF DILATOR STIMULI ON INCREASED MYOGENIC ACTIVATION OF CEREBRAL AND SKELETAL MUSCLE RESISTANCE ARTERIOLES IN OBESE ZUCKER RATS
}

\author{
Joshua T. Butcher ${ }^{1,2}$, Adam G. Goodwill ${ }^{1,2}$, Shyla C. Stanley ${ }^{1,2}$, and Jefferson C. Frisbee ${ }^{1,2}$ \\ ${ }^{1}$ Department of Physiology and Pharmacology, West Virginia University HSC, Morgantown, WV \\ ${ }^{2}$ Center for Cardiovascular and Respiratory Sciences, West Virginia University HSC, \\ Morgantown, WV
}

\section{Abstract}

Objective-To use the obese Zucker rat (OZR) model of the metabolic syndrome to determine the impact of dilator stimuli on myogenic activation (MA) of gracilis arterioles (GA) and middle cerebral arteries (MCA). We tested the hypothesis that increased oxidant stress and thromboxane $\mathrm{A}_{2}\left(\mathrm{TxA}_{2}\right)$ exacerbate MA, and prevent its blunting with dilator stimuli, in OZR.

Methods-GA/MCA from OZR and lean Zucker rats (LZR) were pressurized ex vivo. MA was determined under control conditions and following challenge with acetylcholine, hypoxia and adenosine. Responses were also evaluated after pre-treatment with TEMPOL (antioxidant) and SQ-29548 $\left(\mathrm{PGH}_{2} / \mathrm{TxA}_{2}\right.$ receptor antagonist).

Results-MA was increased (and dilator responses decreased) in GA/MCA from OZR, dependent on the endothelium and ROS. In GA, the impact of ROS on MA and dilator effects was largely via $\mathrm{TxA}_{2}$, while in MCA, this appeared was more dependent on NO bioavailability. Intrinsic responses of GA/MCA to carbacyclin, U46619, and NO donors were similar between strains.

Conclusions-A developing ROS-based endothelial dysfunction in MCA and GA of OZR contributes to an enhanced MA of these vessels. While treatment of GA/MCA with TEMPOL attenuates MA in OZR, the mechanistic contributors to altered MA, distal to ROS, differ between the two resistance vessels.

\section{Keywords}

regulation of vascular tone; rodent models of metabolic syndrome; peripheral resistance; microcirculation

\section{INTRODUCTION}

The metabolic syndrome, also referred to as insulin resistance syndrome or cardiometabolic syndrome, was fully articulated by Reaven in 1988 (25) but has since been more stringently defined with its continual increases in both incidence and prevalence $(1,4,6,7)$. According to the International Diabetes Federation (IDF), a clinical diagnosis of metabolic syndrome is made in patients that have central (abdominal) obesity, and two of the following co-

Address for Correspondence: Jefferson C. Frisbee, Ph.D. Center for Cardiovascular and Respiratory Sciences Department of Physiology and Pharmacology West Virginia University Health Sciences Center; 3152 HSN 1 Medical Center Drive Morgantown, WV 26506 Phone: (304) 293-6527 Fax: (304) 293-5513 jefrisbee@ hsc.wvu.edu. 
morbidities: dyslipidemia, hypertension and impaired glycemic control (1). With these systemic pathologies, a companion pro-inflammatory and pro-thrombotic state is also commonly present $(16,20,22)$. The most significant health outcome of these states is that they severely increase the risk for developing peripheral vascular disease (PVD) $(4,16,20)$, a condition characterized by an inability of the vasculature to effectively match blood perfusion with local tissue/organ metabolic demand. As the regulation of vascular tone and the ability to effectively integrate multiple vasoactive processes is a critical component of blood flow regulation, alterations to the normal patterns of vascular reactivity could severely compromise perfusion:demand matching.

The obese Zucker rat (OZR: fa/fa) is an excellent model for the development of the metabolic syndrome and the associated negative vascular outcomes in humans. It possesses a recessive mutation leading to leptin receptor dysfunction (3, 29), causing a chronic hyperphagic state (17), which results in rapid-onset obesity. By 17 weeks of age, the OZR is severely obese, with the combined presentation of profound insulin resistance, dyslipidemia, and moderate hypertension $(17,26)$; a combination of pathologies that parallels metabolic syndrome in humans.

Full manifestation of the metabolic syndrome in OZR has been well documented to be associated with significant alterations to vascular reactivity $(8,15,28)$. It has previously been shown that myogenic activation of both cerebral (24) and skeletal muscle (10) resistance vessels is enhanced, although there is no consistent understanding of the mechanistic underpinnings of this elevated responsiveness to intralumenal pressure. However, in ex vivo skeletal muscle and cerebral resistance arteries/arterioles from OZR, there is an impaired response to multiple endothelium-dependent dilator stimuli $(8,9,24$, 28 ), and elevated levels of vascular oxidant stress can cause an increased production of the potent vasoconstrictor metabolite thromboxane $\mathrm{A}_{2}\left(\mathrm{TxA}_{2}\right.$; refs: $\left.15,18,21,28\right)$. The extent to which impaired dilator responses and elevated production of constrictor metabolites can impact myogenic activation remains unclear.

Our previous work has suggested that the enhanced myogenic activation of skeletal muscle and cerebral resistance vessels in OZR may not reflect a common mechanistic basis (10,24). Further, it is entirely unclear how myogenic activation in these critically important sites of vascular resistance integrates stimuli from intralumenal pressure and dilator influences to produce a change in diameter. As such, the general purpose of the present study is to determine the fundamental mechanisms of altered myogenic activation between these vascular beds, how this may be impacted by challenge with dilator stimuli, and if identified alterations are due to a consistent, system-wide effect. The general hypothesis is that oxidant stress-based increases in generation of vascular thromboxane $\mathrm{A}_{2}$ will contribute to enhanced myogenic activation and blunted endothelium-dependent dilation in both cerebral and skeletal muscle resistance vessels.

\section{MATERIALS AND METHODS}

\section{Animals}

Male lean Zucker (LZR; n=32) and obese (OZR; n=35) rats (Harlan, Indianapolis, IN) of $\sim 17$ weeks age were used for all experiments. Animals were housed and fed standard chow and tap water ad libitum at the West Virginia University HSC and all protocols received prior approval from the Institutional Animal Care and Use Committee. Table 1 summarizes the baseline characteristics of animals used in the present study and clearly demonstrates the systemic pathologies associated with the metabolic syndrome. Following an overnight fast, all animals were anesthetized with pentobarbital sodium $(50 \mathrm{mg} / \mathrm{kg}$; i.p.) and received tracheal intubation to facilitate maintenance of a patent airway. All animals also received 
cannulation of both a carotid artery for blood pressure recording and a jugular vein for infusion of supplemental anesthetic (as needed) and the collection of blood samples. Venous blood samples were analyzed for glucose (Freestyle Lite, Abbott, Alameda, CA), while the plasma components were analyzed for insulin concentrations (Millipore; Billerica, MA) as well as cholesterol/triglyceride levels (Wako Diagnostics; Richmond, VA), and nitrotyrosine (Oxis International; Foster City, CA). Unless otherwise noted, all drugs and chemicals were purchased from Sigma-Aldrich (St. Louis, MO, USA).

\section{Preparation of Isolated Microvessels}

After the initial surgery (above), a gracilis muscle resistance arteriole (GA) was surgically removed from the anesthetized animal and prepared for video microscopy, as described previously (5). Briefly, the arteriole was placed in a heated $\left(37^{\circ} \mathrm{C}\right)$ bath, doubly-cannulated with glass micropipettes and secured with silk suture. The vessel was then perfused and superfused with a physiological salt solution (PSS) equilibrated with $21 \% \mathrm{O}_{2}, 5 \% \mathrm{CO}_{2}$, balance $\mathrm{N}_{2}$. Immediately following removal of the GA from the animal, the rat was humanely euthanized and the brain was rapidly removed from the skull case, placed in chilled PSS $\left(\sim 4^{\circ} \mathrm{C}\right)$, and the middle cerebral artery (MCA) was surgically removed from the base of the brain. The MCA was then doubly-cannulated and prepared in an identical manner as described for the GA. Both the MCA and the GA were allowed to equilibrate for at least 45 minutes at an intralumenal pressure of $\sim 80 \%$ of the individual animal's mean arterial pressure (equilibration pressure). Vessel dimensions under control conditions and in response to imposed challenge were obtained under pressurized, zero flow conditions using video microscopy and an on-screen video-micrometer (5).

Changes in vessel diameter (myogenic activation) were measured following random changes in intralumenal pressure between $60 \mathrm{mmHg}$ and $140 \mathrm{mmHg}$ in $20 \mathrm{mmHg}$ increments. After the initial assessment of myogenic responses, dilator reactivity was assessed at each level of intralumenal pressure following challenge with hypoxia (20 minutes), acetylcholine $\left(10^{-6}\right.$ $\mathrm{M})$, and adenosine $\left(10^{-6} \mathrm{M}\right)$. For the purposes of the present study, hypoxia was defined as a change in $\mathrm{PO}_{2}$ of the superfusate/perfusate PSS from $135 \mathrm{mmHg}\left[21 \% \mathrm{O}_{2}, 5 \% \mathrm{CO}_{2}\right.$, balance $\mathrm{N}_{2}$ in the equilibration gas] to $\sim 45 \mathrm{mmHg}\left[0 \% \mathrm{O}_{2}, 5 \% \mathrm{CO}_{2}\right.$, balance $\mathrm{N}_{2}$ in the equilibration gas]). These oxygen pressures have been previously validated with $\mathrm{O}_{2}$ microelectrodes in the vessel chamber (19). Upon completion of data collection under untreated control conditions (above), both MCA and GA were treated with either the antioxidant TEMPOL $\left(10^{-4} \mathrm{M}\right)$ or the $\mathrm{PGH}_{2} / \mathrm{TxA}_{2}$ receptor antagonist SQ-29548 $\left(10^{-5} \mathrm{M}\right)$ and at least 30 minutes was allowed for incubation. Following the incubation period, myogenic activation and dilator reactivity to the three stimuli at each level of intralumenal pressure was determined as described above. In an additional series of experiments, following the determination of responses under control conditions, the endothelium of both MCA and GA was denuded by passage of air boli through the vessel lumen (19) and the mechanical responses to intralumenal pressure and dilator stimuli were repeated.

A final series of experiments was performed to determine the responses of isolated GA and MCA from both LZR and OZR, at the equilibration pressures, in response to increasing concentrations of carbacyclin $\left(10^{-12}-10^{-8} \mathrm{M}\right.$; a stable $\mathrm{PGI}_{2}$ analog; Enzo Life Sciences, Farmingdale, NY), U46619 $\left(10^{-12}-10^{-8} \mathrm{M}\right.$; a stable TxA 2 analog; Enzo Life Sciences, Farmingdale, NY), and sodium nitroprusside $\left(10^{-10}-10^{-6} \mathrm{M}\right)$. These experiments were performed to determine if the development of the metabolic syndrome had a significant impact on the intrinsic responses of the GA or MCA in response to the major relevant vasoactive metabolites under interrogation in the present study. 


\section{Data and Statistical Analysis}

All data are presented as mean \pm SE. Active tone of individual vessels at the equilibration pressure was calculated as $\left(\Delta \mathrm{D} / \mathrm{D}_{\max }\right) \bullet 100$, where $\Delta \mathrm{D}$ is the diameter increase from rest in response to $\mathrm{Ca}^{2+}$-free PSS, and $\mathrm{D}_{\max }$ is the maximum diameter measured at the equilibration pressure in $\mathrm{Ca}^{2+}$-free PSS. Curves describing myogenic activation under any of the imposed conditions were fit with linear regression equations: $y=\alpha_{0}+\beta x$; where $y=$ vessel diameter $(\mu \mathrm{m}), x=$ intralumenal pressure $(\mathrm{mmHg}), a_{0}=\mathrm{y}$-intercept, and $\beta$-the slope of myogenic activation of the vessel. All other statistical analyses utilized Student's t-test or analysis of variance (ANOVA) with Student-Newman-Keuls test post-hoc. Statistical significance is represented by $\mathrm{p}<0.05$.

\section{RESULTS}

The baseline characteristics of LZR and OZR used in the present study are summarized in Table 1. At 17 weeks of age, OZR demonstrate significant increases in body mass, moderately elevated blood pressure, increased insulin resistance, and systemic oxidant stress. Table 2 summarizes the diameters of the vessels in this study from both LZR and $\mathrm{OZR}$, at the equilibration pressures, under the different treatment groups in the present study. In GA, there were few differences between LZR and OZR, although the passive diameter of the vessel at the equilibration pressure was reduced in the obese animals. In contrast, MCAs manifested a significant increase in basal tone at the equilibration pressure, but exhibited no reduction in passive diameter.

Figure 1 summarizes myogenic activation of isolated GA (Panel A) and MCA (Panel B) from LZR and OZR in response to elevated intralumenal pressure. In both GA and MCA, the pressure-induced vasoconstriction was significantly elevated in vessels from OZR as compared to responses in LZR. The impact of endothelium denudation is presented in Panels C (for GA) and D (for MCA). Removal of the vascular endothelium abolished almost all of the differences in myogenic activation of either GA or MCA between LZR and OZR.

The impact of pre-treatment of vessels with the antioxidant TEMPOL or the $\mathrm{PGH}_{2} / \mathrm{TxA}_{2}$ receptor antagonist SQ-29548 on myogenic activation is presented in Figure 2. In GA from OZR, pretreatment with either TEMPOL (Panel A) or SQ-29548 (Panel B) significantly reduced the slope of the myogenic activation curve as compared to responses in untreated control conditions. In contrast, while TEMPOL exerted a similar effect on myogenic activation in MCA of OZR as compared to that in GA (Panel C), treatment with SQ-29548 did not significantly impact myogenic activation (Panel D). Combined treatment with both TEMPOL and SQ-29548 did not significantly reduce the slope of myogenic activation beyond that determined for either agent alone (data not shown).

The impact of hypoxia on myogenic activation of GA and MCA is summarized in Figure 3. In GA from LZR, imposition of hypoxia increased arteriolar diameter at the equilibration pressure and blunted myogenic activation. In contrast, challenge with hypoxia had no significant impact on myogenic activation of GA from OZR (Panel A). Pre-treatment with either TEMPOL (Panel B) or SQ-29548 (Panel C) altered this response in GA from OZR, causing not only an increased diameter at the equilibration pressure, but also a significant reduction (i.e., less negative) in the $\beta$ coefficient describing the pressure-induced constrictor response. Combined treatment with both TEMPOL and SQ-29548 did not result in any significant additive effect beyond that determined for either application alone (data not shown). The impact of hypoxia alone (Panel D) and hypoxia following pre-treatment with TEMPOL (Panel E) on myogenic activation of MCA were similar to that for GA from OZR. Hypoxia alone did not significantly impact the enhanced pressure-induced constriction from MCA in OZR, although pre-treatment with TEMPOL not only increased the vessel diameter 
at the equilibration pressure, it also blunted to slope of the myogenic activation curve to levels that were comparable to that in LZR. In contrast, pre-treatment with SQ-29548 did not significantly impact the relationship of myogenic activation and hypoxia in MCA from OZR (Panel F). As with GA, treatment of MCA with both TEMPOL and SQ-29548 did not alter the response as compared to the maximum improvement following single treatment (data not shown).

The impact of acetylcholine on myogenic activation of GA and MCA from LZR and OZR is presented in Figure 4. In GA from OZR, treatment with acetylcholine resulted in a modest dilation at the equilibration pressure, but did not significantly impact the slope of the myogenic activation curve (Panel A). While pre-treatment of GA from OZR with TEMPOL resulted in both an increased dilation at the equilibration pressure and a shifted slope coefficient for myogenic activation to levels that were not different from that in LZR (Panel B), treatment with SQ-29548 (Panel C) demonstrated no identifiable improvements to either the diameter at the equilibration pressure or the slope of myogenic activation. In MCA, acetylcholine increased the vessel diameter at the equilibration pressure in LZR, while also causing a significant shift in slope. In contrast, challenge with acetylcholine resulted in directionally similar, although less striking effect on myogenic activation of MCA from OZR (Panel D). Pre-treatment of MCA from OZR with TEMPOL (+acetylcholine) reduced the severity of myogenic activation to a slope coefficient that was actually more positive than that determined under control conditions in LZR (Panel E). Treatment of MCA from OZR with SQ-29548 (Panel F) did not elicit a significant impact on myogenic activation following treatment with acetylcholine beyond that identified in for acetylcholine treatment alone (Panel D).

The mechanical responses of GA and MCA from LZR and OZR to increasing concentration of carbacyclin (Panel A), U46619 (Panel B) and sodium nitroprusside (Panel C) are summarized in Figure 5. For carbacyclin and sodium nitroprusside, dilator responses of GA and MCA in response to increasing concentrations of the agonist were brisk throughout the concentration range, with no significant differences between vessels from LZR and OZR. Similarly, constrictor responses to U46619, while brisk and extensive for both GA and MCA were not significantly different between LZR and OZR.

Figure 6 presents myogenic activation of GA and MCA from LZR and OZR in response to the presence of elevated adenosine $\left(10^{-6} \mathrm{M}\right)$. In both GA (Panel A) and MCA (Panel B), the presence of adenosine significantly increased vessel diameter at the respective equilibration pressures and blunted the severity of the enhanced myogenic activation.

\section{DISCUSSION}

Systemic PVD is an insidious disease that is characterized by an inability of the tissue/organ vasculature to adequately match local metabolic demand with perfusion. While there can be several contributors to this outcome, alterations to the control of vascular tone within resistance vessels represents a dominant factor. The OZR has proven to be an excellent model for the human progression to non-atherosclerotic PVD as the metabolic syndrome can alter vascular reactivity in the absence of plaque/lesion development (2). However, one of the challenges of studying any disease with a functional outcome on the regulation of blood flow and its intra-organ distribution is an understanding of how resistance arteries/arterioles integrate multiple inputs to produce an appropriate response. It has been established that OZR possess an enhanced myogenic activation in response to intralumenal pressure in both the skeletal muscle (10) and cerebral circulation (24). It has also been demonstrated that the chronic increase in vascular oxidant stress in OZR results in a shift in arachidonic acid metabolism, increasing production of vascular $\mathrm{TxA}_{2}$ over levels determined in LZR (15). 
Further, it has been demonstrated that this this process can have a marked deleterious impact on intra-organ distribution of perfusion via alterations to the integrated regulation of vascular tone in skeletal muscle (11). From this, the question arises as to how vascular myogenic activation within the metabolic syndrome is impacted by changes in dilator stimuli and whether interventions targeting elevated oxidant stress or elevated $\mathrm{TxA}_{2}$ production are effective at restoring normal patterns of reactivity between myogenic activation and interactions with dilator influences.

The initial observation from this study is that pressure-induced constriction is increased in both GA and MCA from OZR as compared to LZR, supporting previous observations (10, 24). Removal of the vascular endothelium largely abolished differences in myogenic activation between OZR and LZR, suggesting that the endothelium may represent the primary contributor to differences between the strains. Previous evidence using OZR $(9,10$, $15,28)$ and similar models (2) suggests that two endothelium-dependent mechanisms, both of which stem from a chronically elevated vascular oxidant stress, may be responsible for the endothelial influence on myogenic activation: a reduction to vascular NO bioavailability via increased ROS scavenging and a shift in arachidonic acid metabolism toward production of the vasoconstrictor $\mathrm{TxA}_{2}$. This interpretation is supported by data presented in Figure 2, where pre-treatment of GA or MCA of OZR with the TEMPOL shifted myogenic activation to levels that were comparable to those determined in LZR. However, while is it clear that ROS plays a role in myogenic activation of both GA and MCA, the role for $\mathrm{TxA}_{2}$ is less consistent. In GA, pre-treatment with SQ-29548 resulted in a similar effect to that for TEMPOL, a restoration of pressure-induced constriction to levels in LZR. However, this treatment was largely without effect in MCA from OZR (Figure 2, Panel D). These data suggest that the contribution of ROS to increased myogenic activation may not be consistent across vascular beds; being more dependent on $\mathrm{TxA}_{2}$ in skeletal muscle arterioles, and more dependent on NO bioavailability in the cerebral circulation.

To further explore the potential contribution of altered arachidonic acid metabolism to the main observations, the myogenic activation of ex vivo GA and MCA was evaluated under conditions of hypoxia. While other contributors to this response have been identified, including $\mathrm{NO}(8,13,24)$ and metabolites produced from cytochrome $\mathrm{P} 450$ whydroxlases and epoxygenases $(8,12,13)$, in both GA and MCA, this has been demonstrated to be a dilator response that is largely-dependent on the production of $\mathrm{PGI}_{2}$ from arachidonic acid $(15,19,21)$. As shown in Figure 3, imposition of hypoxia alone had only mild effects on myogenic activation in either GA or MCA from OZR. This was not surprising, as hypoxic dilation of resistance vessels is impaired in OZR, in large measure due to a reduction in mediator bioavailability and an increased production of $\operatorname{TxA}_{2}(8,15,24)$. However, when pre-treated with TEMPOL, the impact of hypoxia became much more pronounced, significantly blunting the slope of myogenic activation in vessels from OZR. This observation clearly suggests that the increased vascular oxidant stress is contributing to not just the enhanced myogenic activation of GA and MCA, but also to the inability of hypoxia to blunt the enhanced pressure-induced constriction in vessels from OZR. An intriguing disparity exists when this is extended to the potential role for $\mathrm{TxA}_{2}$, as pre-treatment with SQ-29548 resulted in an identical outcome as that for TEMPOL pre-treatment in GA from OZR (Figure 3, Panel C), while having a very limited effect in MCA (Figure 3, Panel F). These data clearly suggest that the impact of ROS on myogenic activation in GA from OZR, and the relative inability of hypoxia to blunt the pressure-induced constriction in these vessels, may be mediated predominantly through the production and actions of $\mathrm{TxA}_{2}$. In contrast, while the effects of ROS and the impact of hypoxia on myogenic activation of MCA from OZR are qualitatively similar in MCA, the contribution of $\mathrm{TxA}_{2}$ to this process may be considerably lower. 
Pursuant to this, we switched to a mechanistically different dilator, acetylcholine, which is largely NO-dependent in the rat vasculature (27). While the impact of acetylcholine on myogenic activation of GA and MCA from OZR was comparable to that for hypoxia, and pre-treatment with TEMPOL increased the impact of the stimulus on myogenic activation of both GA and MCA, pre-treatment with SQ-29548 had no identifiable impact on myogenic activation of either vessel (+ acetylcholine; Figure 4, Panels $C$ and F). Based on previous data regarding the mechanistic pathways for acetylcholine and the results presented above, these data provide additional support for the concept of a divergence in ROS-dependent contributors to the enhanced myogenic activation of GA and MCA from OZR.

One question that arises from the results of the present study is the intrinsic sensitivity of GA and MCA from LZR and OZR in response to $\mathrm{PGI}_{2}$ (carbacyclin), TxA 2 (U46619) and nitric oxide, and whether this changes with the evolution of the metabolic syndrome. However, the data presented in Figure 5 indicate that vascular responsiveness to these three metabolites remains robust and does not demonstrate any consistent alteration in this model. As such, it is plausible at this time to ascribe differences in vascular responses to myogenic stimulation and dilator influences as an issue of mediator bioavailability, rather than one of vascular responsiveness.

Used as a proxy variable for elevated metabolic demand, the impact of elevated adenosine $\left(10^{-6} \mathrm{M}\right)$ on myogenic activation of GA and MCA from OZR reveals an intriguing relationship. In skeletal muscle arteriole of OZR, elevated adenosine attenuated the severity of the pressure-induced vasoconstriction, resulting in a response that was similar to that determined in GA from LZR. One would predict that this would be an appropriate response of the resistance vasculature in response to elevated metabolic demand - a shift in the overall setting of myogenic activation, such that flow regulation can still be maintained, although at a lower overall resistance. However, given that this 'adenosine-treated' response remains blunted below that in GA from LZR treated with adenosine, altered myogenic activation in GA of OZR may still represent a contributor to reduced bulk perfusion and altered perfusion distribution with elevated metabolic demand (14). In contrast, application of adenosine to MCA resulted in a very comparable blunting of myogenic activation in vessels from both LZR and OZR. Speculatively, these observations suggest that the ability of local metabolic demand in the brain may be more able to override the increased pressureinduced constriction in the cerebral resistance vessels of OZR than in the skeletal muscle microcirculation. However, the extent to which this may serve as a protective mechanism under conditions of cerebral ischemia or hypoxia in OZR, where stroke severity may be enhanced (22) remains unknown at this time.

\section{PERSPECTIVES}

With the development of the metabolic syndrome in this model, an increased pressureinduced constriction of resistance vessels develops in both the skeletal muscle and cerebral circulation. While the genesis of this increased myogenic activation for both vessels appears to lie within an elevation in vascular oxidant stress, the increased response in skeletal muscle vessels appears to reflect an increased contribution from the production of $\mathrm{TxA}_{2}$, while responses in the cerebral circulation appear to reflect a loss of dilator metabolite bioavailability. Importantly, the ability of local metabolic demand to override myogenic activation appears to still be present in both tissues, and may be fully intact in the brain. While this may reflect a protective mechanism in the brain versus skeletal muscle, further interrogation into the functional impact of these differences on organ perfusion as well as therapeutic interventions against the increased myogenic activation should keep this in mind. 


\section{Acknowledgments}

This study was supported by grants from the National Institutes of Health (NIH T32 HL 90610, RR 2865AR and IDeA P20 GM 103434), the American Heart Association (AHA EIA 0740129N). The authors also wish to express their gratitude for the expert technical assistance from Ms. Milinda James from the Department of Physiology and Pharmacology at West Virginia University.

\section{List of Abbreviations}

$\begin{array}{ll}\text { GA } & \text { Gracilis Arteriole } \\ \text { MCA } & \text { Middle Cerebral Artery } \\ \text { TxA2 } & \text { Thromboxane } A_{2} \\ \text { ROS } & \text { Reactive Oxygen Species }\end{array}$

\section{LITERATURE CITED}

1. Alberti KG, Zimmet P, Shaw J. Metabolic syndrome--a new world-wide definition. A Consensus Statement from the International Diabetes Federation. Diabet Med. 2006; 23:469-480. [PubMed: 16681555]

2. Amy RM, Dolphin PJ, Pederson RA, Russell JC. Atherogenesis in two strains of obese rats: The fatty Zucker and LA/N-corpulent. Atherosclerosis. 1998; 69:199-209. [PubMed: 3348841]

3. Bray GA. The Zucker-fatty rat: a review. Fed Proc. 1977; 36:148-153. [PubMed: 320051]

4. Bray GA, Bellanger T. Epidemiology, trends, and morbidities of obesity and the metabolic syndrome. Endocrine. 2006; 29:109-117. [PubMed: 16622298]

5. Butcher JT, Goodwill AG, Frisbee JC. The ex vivo isolated skeletal microvessel preparation for investigation of vascular reactivity. J Vis Exp. 28:2012.

6. Eckel RH, Grundy SM, Zimmet PZ. The metabolic syndrome. Lancet. 2005; 365:1415-1428. [PubMed: 15836891]

7. Ervin RB. Prevalence of metabolic syndrome among adults 20 years of age and over, by sex, age, race and ethnicity, and body mass index: United States, 2003-2006. Natl Health Stat Report. 2009; 13:1-7. [PubMed: 19634296]

8. Frisbee JC. Impaired dilation of skeletal muscle microvessels to reduced oxygen tension in diabetic obese Zucker rats. Am J Physiol Heart Circ Physiol. 2001; 281:H1568-H1574. [PubMed: 11557545]

9. Frisbee JC. Reduced nitric oxide bioavailability contributes to skeletal muscle microvessel rarefaction in the metabolic syndrome. Am J Physiol Regul Integr Comp Physiol. 2005; 289:R307R316. [PubMed: 15802560]

10. Frisbee JC, Maier KG, Stepp DW. Oxidant stress-induced increase in myogenic activation of skeletal muscle resistance arteries in obese Zucker rats. Am J Physiol Heart Circ Physiol. 2002; 283:H2160-H2168. [PubMed: 12388303]

11. Frisbee JC, Hollander JM, Brock RW, Yu HG, Boegehold MA. Integration of skeletal muscle resistance arteriolar reactivity for perfusion responses in the metabolic syndrome. Am J Physiol Regul Integr Comp Physiol. 2009; 296:R1771-R1782. [PubMed: 19386988]

12. Frisbee JC, Krishna UM, Falck JR, Lombard JH. Role of prostanoids and 20-HETE in mediating oxygen-induced constriction of skeletal muscle resistance arteries. Microvascular Research. 2001; 62:271-283. [PubMed: 11678630]

13. Frisbee JC, Maier KG, Falck JR, Roman RJ, Lombard JH. Integration of hypoxic dilation signaling pathways for skeletal muscle resistance arteries. Am J Phys Reg Int and Comp Physiology. 2002; 283:R309-R319.

14. Frisbee JC, Goodwill AG, Butcher JT, Olfert IM. Divergence between arterial perfusion and fatigue resistance in skeletal muscle in the metabolic syndrome. Exp Physiol. 2011; 96:369-383. [PubMed: 21123363] 
15. Goodwill AG, James ME, Frisbee JC. Increased vascular thromboxane generation impairs dilation of skeletal muscle arterioles of obese Zucker rats with reduced oxygen tension. Am J Physiol Heart Circ Physiol. 2008; 295:H1523-H1528.

16. Gorter PM, Olijoek JK, Van Der GY, Algra A, Rabelink TJ, Visseren FL. Prevalence of the metabolic syndrome in patients with coronary heart disease, cerebrovascular disease, peripheral arterial disease or abdominal aortic aneurysm. Atherosclerosis. 2004; 173:363-369. [PubMed: 15064114]

17. Kurtz TW, Morris RC, Pershadsingh HA. The Zucker fatty rat as a genetic model of obesity and hypertension. Hypertension. 1989; 13:896-901. [PubMed: 2786848]

18. Lenda DM, Sauls BA, Boegehold MA. Reactive oxygen species may contribute to reduced endothelium-dependent dilation in rats fed high salt. Am J Phys Heart and Circulatory Physiology. 2000; 279:H7-H14.

19. Lombard JH, Liu Y, Fredricks KT, Bizub DM, Roman RJ, Rusch NJ. Electrical and mechanical responses of rat middle cerebral arteries to reduced $\mathrm{PO}_{2}$ and prostacyclin. Am J Phys Heart and Circ Phys. 1999; 276:H509-H516.

20. Lusis AJ, Attie AD, Reue K. Metabolic syndrome: from epidemiology to systems biology. Nat Rev Genet. 2008; 9:819-830. [PubMed: 18852695]

21. Messina EJ, Sun D, Koller A, Wolin MS, Kaley G. Increases in Oxygen Tension Evoke Arteriolar Constriction by Inhibiting Endothelial Prostaglandin Synthesis. Microvascular Research. 1994; 48:151-160. [PubMed: 7854203]

22. Miranda PJ, Defronzo RA, Califf RM, Guyton JR. Metabolic syndrome: definition, pathophysiology, and mechanisms. Am Heart J. 2005; 149:33-45. [PubMed: 15660032]

23. Osmond JM, Mintz JD, Dalton B, Stepp DW. Obesity increases blood pressure, cerebral vascular remodeling and severity of stroke in the Zucker rat. Hypertension. 2009; 53:381-386. [PubMed: 19104000]

24. Phillips SA, Sylvester FA, Frisbee JC. Oxidant stress and constrictor reactivity impair cerebral artery dilation in obese Zucker rats. Am J Physiol Regul Integr Comp Physiol. 2005; 288:R523R530.

25. Reaven GM. Banting lecture 1988. Role of insulin resistance in human disease. Diabetes. 1988; 37:1595-1607. [PubMed: 3056758]

26. Stepp DW, Frisbee JC. Augmented adrenergic vasoconstriction in hypertensive diabetic obese Zucker rats. Am J Physiol Heart Circ Physiol. 2002; 282:H816-H820. [PubMed: 11834474]

27. Ungvari Z, Sun D, Huang A, Kaley G, Koller A. Role of endothelial [Ca2+] in activation of eNOS in pressurized arterioles by agonists and wall shear stress. Am J Physiol Heart Circ Physiol. 2001; 281:H606-H612. [PubMed: 11454563]

28. Xiang L, Naik JS, Hodnett BL, Hester RL. Altered arachidonic acid metabolism impairs functional vasodilation in metabolic syndrome. Am J Physiol Regul Integr Comp Physiol. 2006; 290:R134R138. [PubMed: 16166209]

29. Zucker LM, Antoniades HN. Insulin and obesity in the Zucker genetically obese rat "fatty". Endocrinology. 1972; 90:1320-1330. [PubMed: 5012744] 

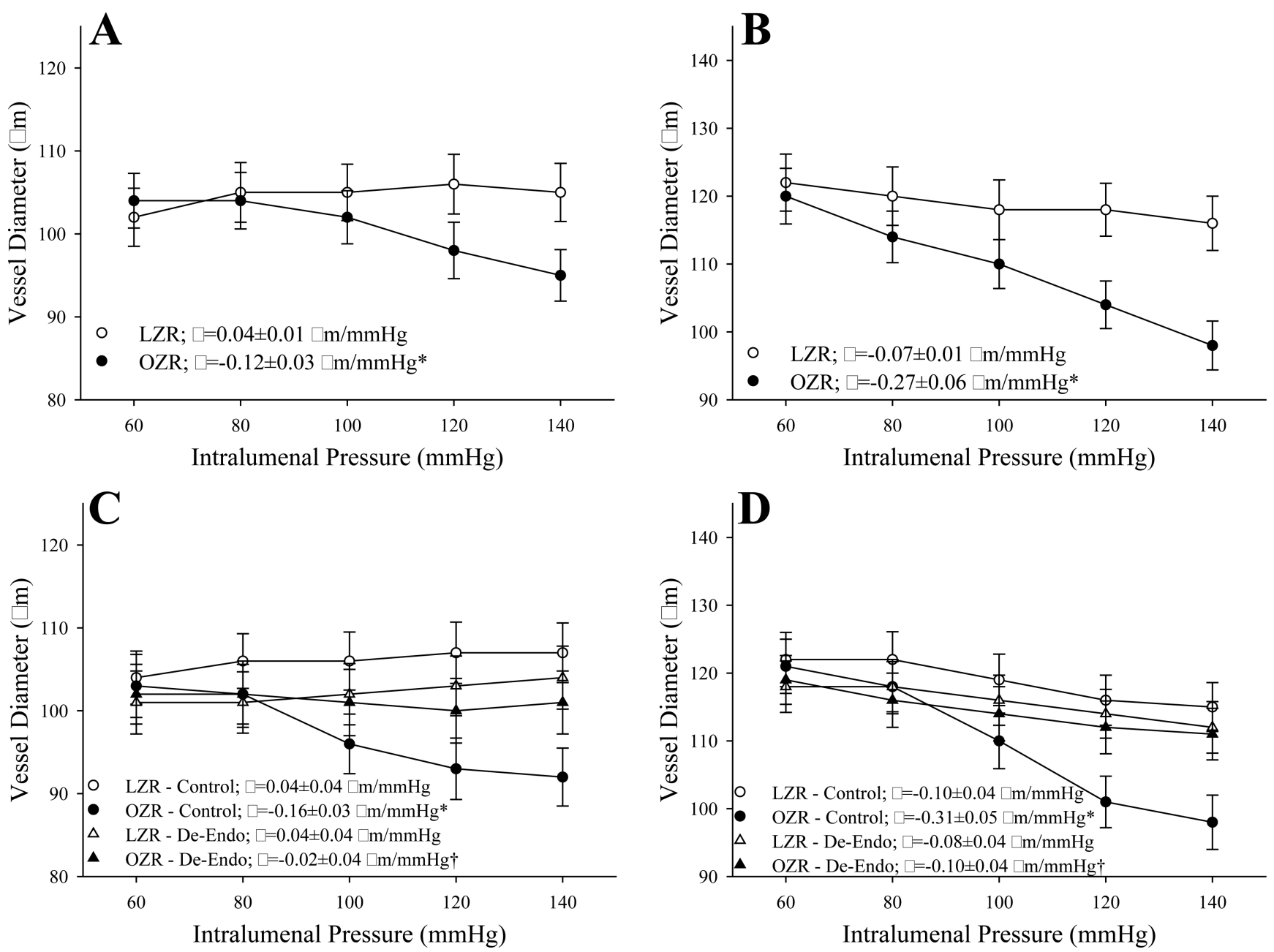

Figure 1.

Data describing myogenic activation of isolated gracilis muscle arterioles (Panels A and C; $\mathrm{n}=5$ for both) and middle cerebral arteries (Panels B and D; $\mathrm{n}=5-6$ for both) from LZR and OZR in response to increased intralumenal pressure. Data, presented as mean $\pm \mathrm{SE}$, are presented for vessels under control conditions (Panels A and B) and following removal of the endothelium (Panels $C$ and D). Slope $(\beta)$ coefficients describing the curve of myogenic activation are presented in the inset legend. $* \mathrm{p}<0.05$ vs. LZR-Control; $\uparrow \mathrm{p}<0.05$ vs.OZRControl. 

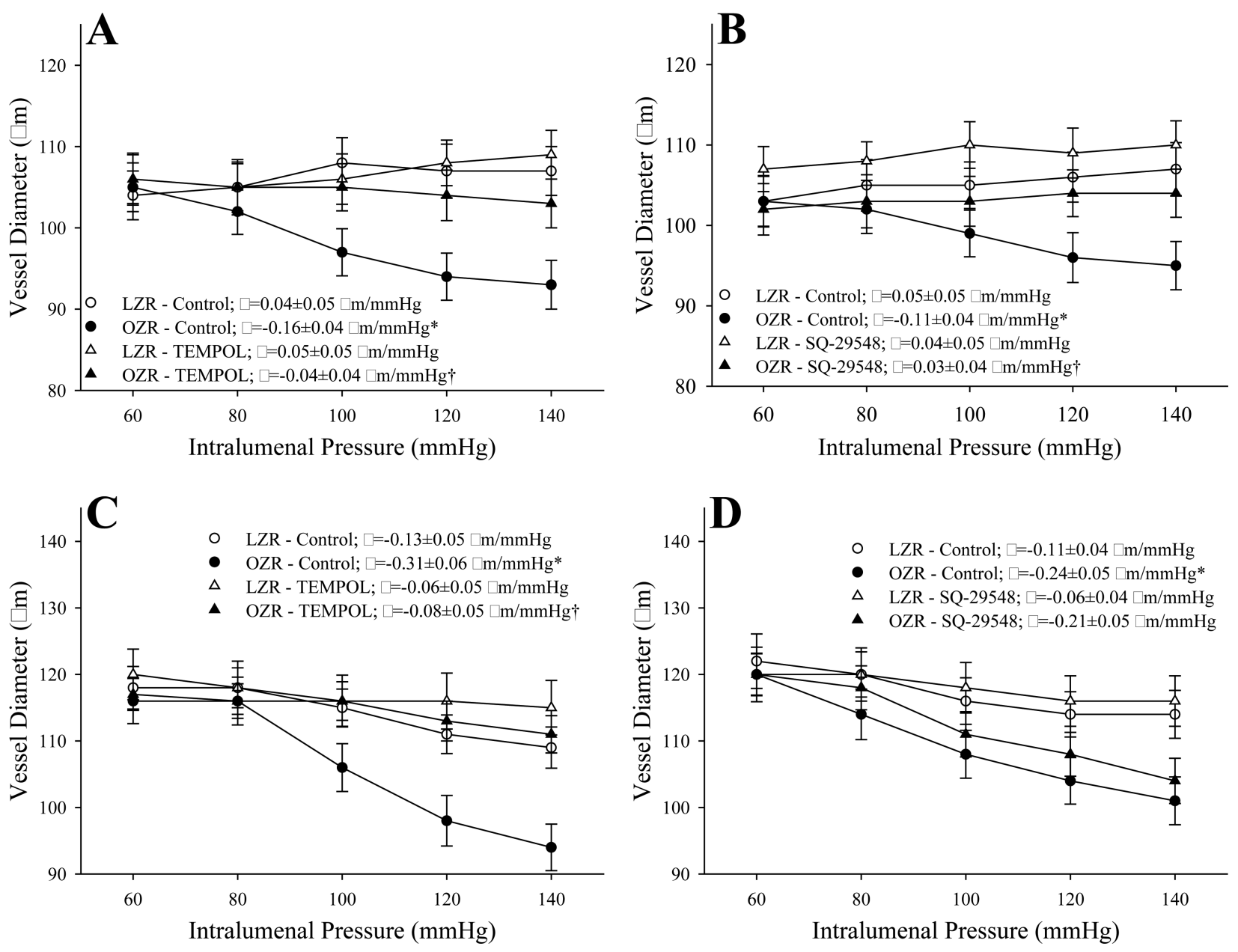

Figure 2.

Data describing myogenic activation of isolated gracilis muscle arterioles (Panels A and B; $\mathrm{n}=5-7$ for both) and middle cerebral arteries (Panels $\mathrm{C}$ and $\mathrm{D} ; \mathrm{n}=6$ for both) from LZR and $\mathrm{OZR}$ in response to increased intralumenal pressure. Data, presented as mean $\pm \mathrm{SE}$, are presented for vessels under control conditions and following pre-treatment of the vessels with either TEMPOL $\left(10^{-4} \mathrm{M}\right)$ or the $\mathrm{PGH}_{2} / \mathrm{TxA}_{2}$ receptor antagonist SQ-29548 $\left(10^{-5} \mathrm{M}\right)$. Slope $(\beta)$ coefficients describing the curve of myogenic activation are presented in the inset legend. $* \mathrm{p}<0.05$ vs. LZR-Control; $\uparrow \mathrm{p}<0.05$ vs.OZR-Control. 

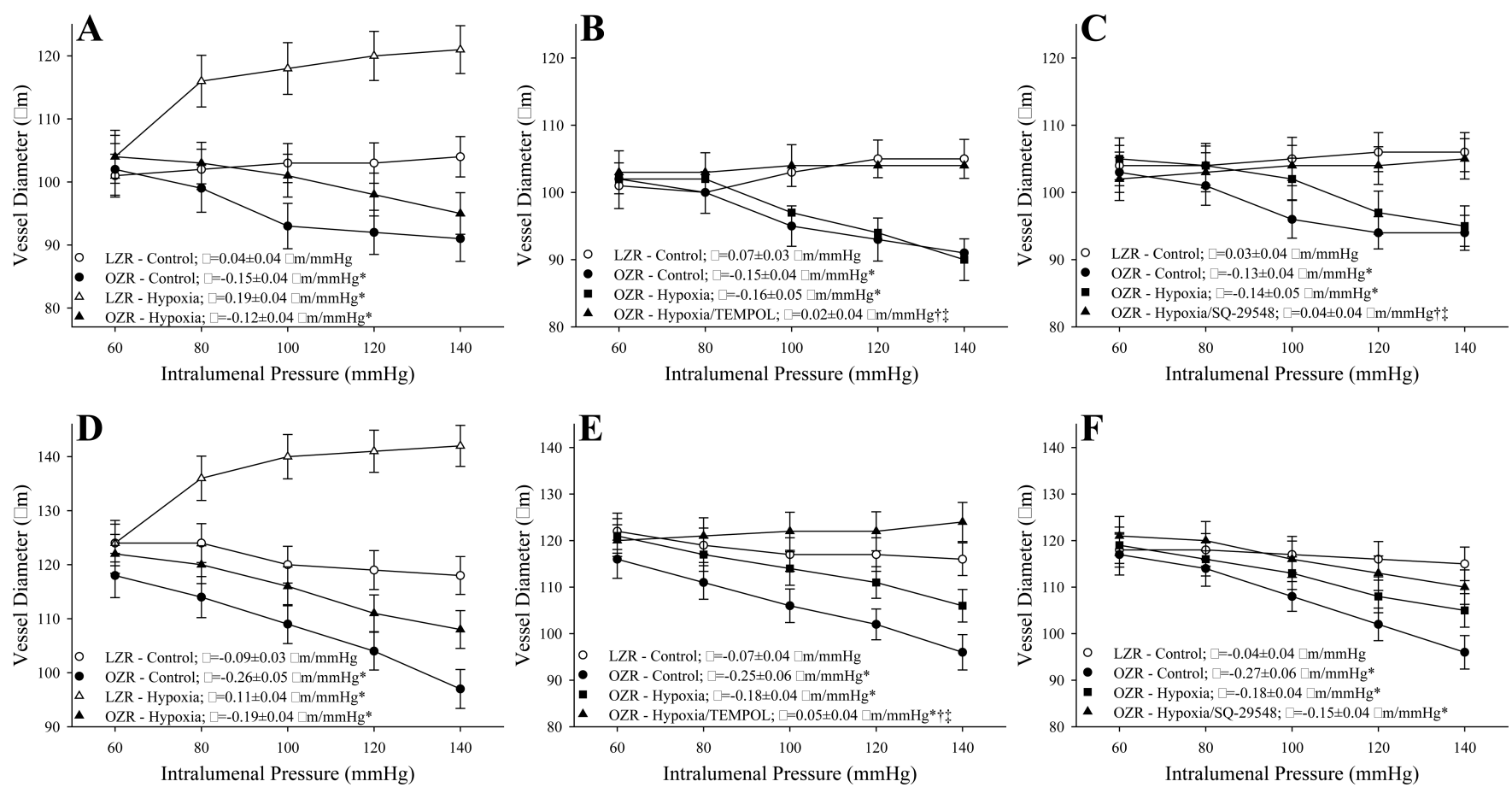

Figure 3.

Data describing myogenic activation of isolated gracilis muscle arterioles (Panels A-C; $\mathrm{n}=5-6$ for all) and middle cerebral arteries (Panels D-F; $\mathrm{n}=5$ for all) from LZR and OZR in response to increased intralumenal pressure. Data, presented as mean $\pm \mathrm{SE}$, are presented for vessels under control conditions, following imposition of hypoxia and following pretreatment of the hypoxic vessels with either TEMPOL $\left(10^{-4} \mathrm{M}\right)$ or the $\mathrm{PGH}_{2} / \mathrm{TxA}_{2}$ receptor antagonist SQ-29548 $\left(10^{-5} \mathrm{M}\right)$. Slope $(\beta)$ coefficients describing the curve of myogenic activation are presented in the inset legend. $* \mathrm{p}<0.05$ vs. LZR-Control; $\uparrow \mathrm{p}<0.05$ vs.OZRControl; $\ddagger \mathrm{p}<0.05$ vs. OZR-Hypoxia. 

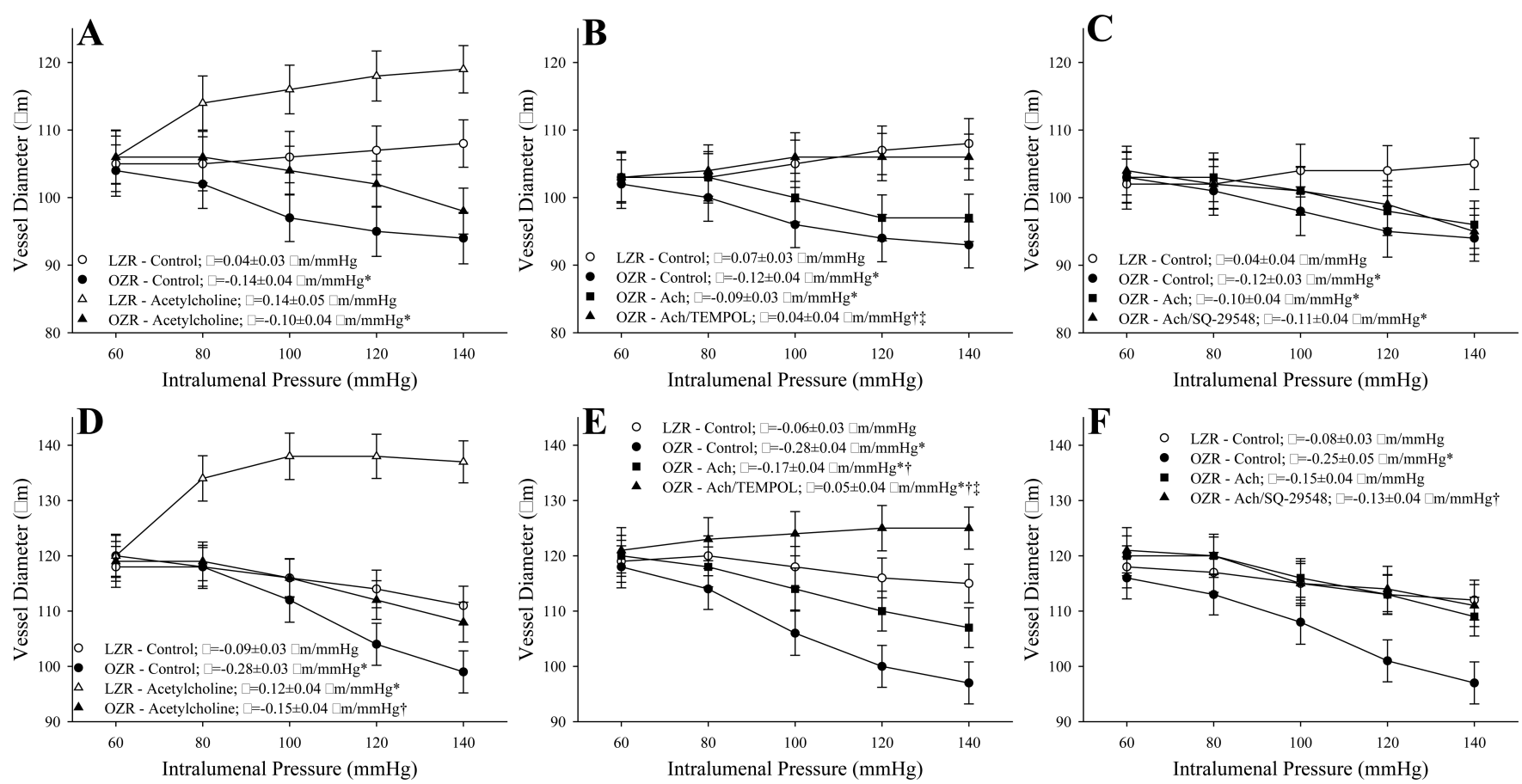

Figure 4.

Data describing myogenic activation of isolated gracilis muscle arterioles (Panels A-C; $\mathrm{n}=5$ for all) and middle cerebral arteries (Panels D-F; $n=5-6$ for all) from LZR and OZR in response to increased intralumenal pressure. Data, presented as mean $\pm \mathrm{SE}$, are presented for vessels under control conditions, following challenge with acetylcholine $\left(10^{-6} \mathrm{M}\right)$ and following pre-treatment of the acetylcholine-challenged vessels with either TEMPOL $\left(10^{-4} \mathrm{M}\right)$ or the $\mathrm{PGH}_{2} / \mathrm{TxA}_{2}$ receptor antagonist SQ-29548 $\left(10^{-5} \mathrm{M}\right)$. Slope $(\beta)$ coefficients describing the curve of myogenic activation are presented in the inset legend. $* \mathrm{p}<0.05$ vs. LZR-Control; $\dagger \mathrm{p}<0.05$ vs.OZR-Control; $\ddagger \mathrm{p}<0.05$ vs. OZR-Hypoxia. 

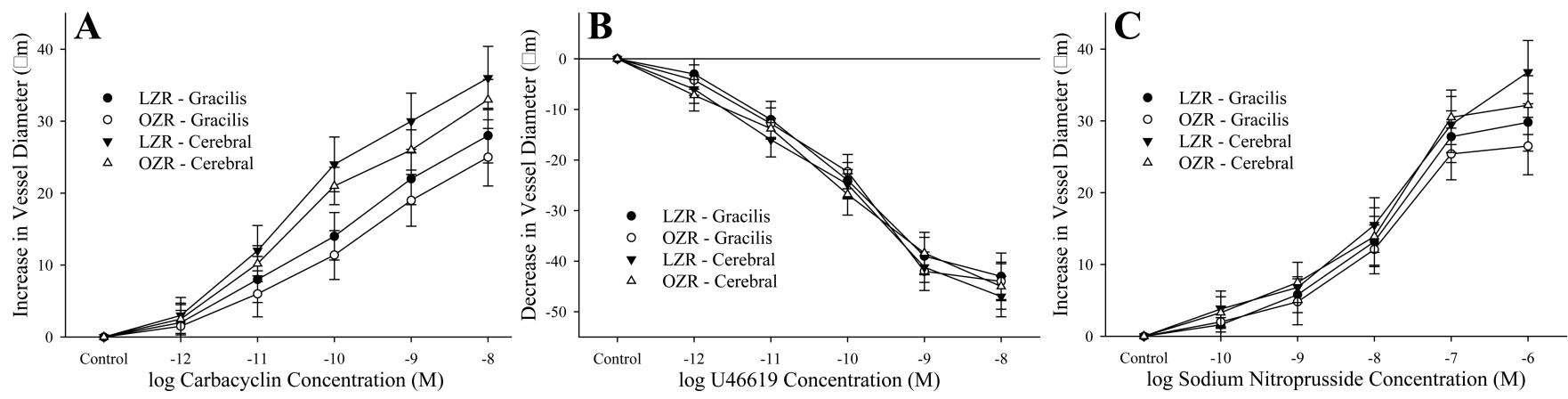

Figure 5.

Data describing the mechanical responses of isolated gracilis muscle arterioles and middle cerebral arteries from LZR and OZR in response to increased concentrations of carbacyclin (Panel A; n=5 for all), U46619 (Panel B; n=5 for all) and sodium nitroprusside (Panel C; $\mathrm{n}=5$ for all). Data, presented as mean $\pm \mathrm{SE}$, are presented for vessels under control conditions, at their equilibrium pressure. There were no significant differences in the mechanical responses of GA or MCA between LZR and OZR for the three agonists. 

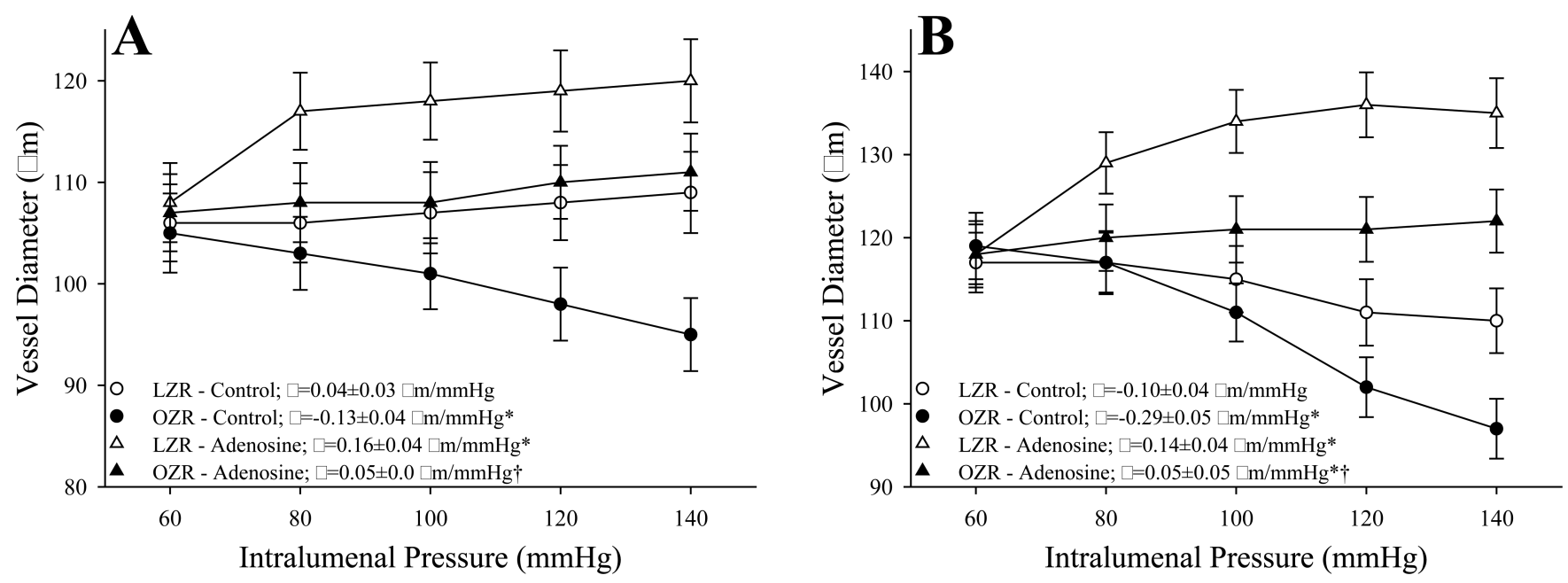

Figure 6.

Data describing myogenic activation of isolated gracilis muscle arterioles (Panel A; $\mathrm{n}=5$ ) and middle cerebral arteries (Panel $\mathrm{B} ; \mathrm{n}=5$ ) from LZR and OZR in response to increased intralumenal pressure. Data, presented as mean $\pm \mathrm{SE}$, are presented for vessels under control conditions and following challenge with adenosine $\left(10^{-6} \mathrm{M}\right) . * \mathrm{p}<0.05$ vs. LZR-Control; $\dagger$ $\mathrm{p}<0.05$ vs.OZR-Control. 
Table 1

Baseline characteristics of 17 week-old LZR and OZR used in the present study.

\begin{tabular}{lcc}
\hline & LZR $(\mathbf{n}=\mathbf{3 0})$ & OZR $(\mathbf{n}=\mathbf{3 4})$ \\
\hline Mass $(\mathrm{g})$ & $344 \pm 11$ & $669 \pm 12^{*}$ \\
MAP (mmHg) & $106 \pm 6$ & $126 \pm 5^{*}$ \\
& $105 \pm 11$ & $181 \pm 10^{*}$ \\
{$[\text { Glucose }]_{\text {plasma }}(\mathrm{mg} / \mathrm{dl})$} & $1.4 \pm 0.2$ & $8.5 \pm 1.2^{*}$ \\
{$[\text { Insulin] }]_{\text {plasma }}(\mathrm{ng} / \mathrm{ml})$} & $15 \pm 5$ & $45 \pm 8{ }^{*}$ \\
{$[\text { Nitrotyrosine }]_{\text {plasma }}(\mathrm{ng} / \mathrm{ml})$} & & \\
\hline * $<0.05$ versus LZR. & &
\end{tabular}




\section{Table 2}

Inner diameter (at the equilibration pressure) of gracilis arterioles (GA) and middle cerebral arteries (MCA) of LZR and OZR used in the present study.

\begin{tabular}{lcccc}
\hline & \multicolumn{2}{c}{ Gracilis Arteriole } & \multicolumn{2}{c}{ Middle Cerebral Artery } \\
\hline & LZR & OZR & LZR & OZR \\
Control & $104 \pm 5(44 \pm 4 \%)$ & $96 \pm 5(43 \pm 3 \%)$ & $121 \pm 4(43 \pm 4 \%)$ & $105 \pm 5(46 \pm 4 \%)^{*}$ \\
-Endothelium & $101 \pm 4$ & $100 \pm 5$ & $118 \pm 5$ & $114 \pm 4$ \\
+ Hypoxia & $118 \pm 4$ & $100 \pm 5$ & $138 \pm 4$ & $115 \pm 5 *$ \\
+ SQ-29548 & $107 \pm 4$ & $102 \pm 4$ & $120 \pm 5$ & $109 \pm 4$ \\
+ TEMPOL & $104 \pm 5$ & $105 \pm 4$ & $116 \pm 5$ & $113 \pm 5$ \\
+ Acetylcholine & $115 \pm 5$ & $101 \pm 5$ & $133 \pm 5$ & $112 \pm 4$ \\
+ Adenosine & $118 \pm 4$ & $109 \pm 5$ & $129 \pm 4$ & $119 \pm 5$ \\
Ca $^{2+}$-free PSS (Passive) & $184 \pm 5$ & $169 \pm 5$ & $204 \pm 4$ & $194 \pm 5$ \\
\hline
\end{tabular}

Data in parentheses represents \% active tone.

p $<<0.05$ versus LZR. 